\title{
1 Developmental regulation of neuronal gene expression by Elongator complex protein 1
}

2 dosage.

4 Elisabetta Morini ${ }^{1,2,8}$, Dadi Gao ${ }^{1,2,3,8}$, Emily M. Logan ${ }^{1}$, Monica Salani ${ }^{1}$, Aram J. Krauson ${ }^{1}$, Anil

5 Chekuri $^{1,2}$, Yei-Tsung Chen ${ }^{4}$, Ashok Ragavendran ${ }^{1,3}$, Probir Chakravarty ${ }^{5}$, Serkan Erdin ${ }^{1,3}$, Alexei

6 Stortchevoi ${ }^{1,3}$, Jesper Q. Svejstrup ${ }^{6,7}$, Michael E. Talkowski ${ }^{1,2,3}$, Susan A. Slaugenhaupt ${ }^{1,2 *}$

$8{ }^{1}$ Center for Genomic Medicine, Massachusetts General Hospital Research Institute, Boston, MA.

$9 \quad{ }^{2}$ Department of Neurology, Massachusetts General Hospital Research Institute and Harvard

10 Medical School, Boston, MA.

$11{ }^{3}$ Program in Medical and Population Genetics and Stanley Center for Psychiatric Research, Broad

12 Institute of Harvard and MIT, Cambridge, MA.

$13{ }^{4}$ Department of Life Sciences and Institute of Genome Sciences, National Yang Ming Chiao Tung

14 University, Taiwan.

$15{ }^{5}$ Bioinformatics and Biostatistics, The Francis Crick Institute, London, UK

$16{ }^{6}$ Mechanisms of Transcription Laboratory, The Francis Crick Institute, London, UK.

$17{ }^{7}$ Department of Cellular and Molecular Medicine, Panum Institute, University of Copenhagen,

18 Copenhagen, Denmark.

$19{ }^{8}$ These authors contributed equally to this work.

$20 *$ Correspondence should be addressed to S.A.S. (slaugenhaupt@,mgh.harvard.edu). 


\section{Abstract}

23 Elongator is a highly conserved protein complex required for transcriptional elongation,

24 intracellular transport and translation. Elongator complex protein 1 (ELP1) is the scaffolding

25 protein of Elongator and is essential for its assembly and stability. Familial dysautonomia (FD), a

26 hereditary sensory and autonomic neuropathy, is caused by a mutation in ELP1 that lead to a tissue-

27 specific reduction of ELP1 protein. Our work to generate a phenotypic mouse model for FD led to

28 the discovery that homozygous deletion of the mouse Elpl gene leads to embryonic lethality prior

29 to mid-gestation. Given that FD is caused by a reduction, not loss, of ELP1, we generated two new

30 mouse models by introducing different copy numbers of the human FD ELP1 transgene into the

31 Elp1 knockout mouse $\left(E l p 1^{-/}\right)$and observed that human ELP1 expression rescues embryonic

32 development in a dose dependent manner. We then conducted a comprehensive transcriptome

33 analysis in mouse embryos to identify genes and pathways whose expression correlates with the

34 amount of ELP1. We found that ELP1 is essential for the expression of genes responsible for the

35 formation and development of the nervous system. Further, gene length analysis of the

36 differentially expressed genes showed that the loss of Elp1 mainly impacts the expression of long

37 genes and that by gradually restoring Elongator their expression is progressively rescued. Finally,

38 through evaluation of co-expression modules, we identified gene sets with unique expression

39 patterns that depended on ELP1 expression. Overall, this study highlights the crucial role of ELP1

40 during early embryonic neuronal development and reveals gene networks and biological pathways

41 that are regulated by Elongator. 


\section{Introduction}

44 Elongator is a highly conserved multiprotein complex composed of two copies of each of its six

45 subunits, named Elongator complex proteins 1 to 6 (ELP1-6). Elongator subunits are

46 evolutionarily highly conserved from yeast to humans both in their sequence and interaction with

47 other subunits. Conserved function across all species has been clearly demonstrated using a variety

48 of different cross-species rescue experiments (1-3). Deletion of any of the genes encoding the six

49 subunits confers almost identical biochemical phenotypes in yeast (4-6), suggesting that there is a

50 tight functional association between the proteins comprising Elongator complex, and that the

51 functional integrity of Elongator is compromised in the absence of any of its subunits (6). Both

52 yeast and human Elongator have lysine acetyltransferase activity that is mediated by the catalytic

53 subunit Elp3. Elp3 has two identified substrates: histone H3 and $\alpha$-Tubulin (7-10). While the

54 acetylation of histone $\mathrm{H} 3$ is linked to the role of the complex in transcriptional elongation $(7,10$,

55 11), cytosolic acetylation of $\alpha$-Tubulin has been linked to its role in microtubule organization

56 particularly in the context of cell migration (8). Elongator was isolated as a complex that associates

57 with chromatin and interacts with the elongating phosphorylated form of RNA polymerase II

58 (RNAPII) both in yeast and human $(7,10,12)$. The catalytic subunit Elp3, by acetylating histone

$59 \mathrm{H} 3$, facilitates RNAPII access to actively transcribed genes. In human cells, Elongator is required

60 for the expression of several genes involved in migration and in the expression of HSP70 $(13,14)$.

61 In addition, accumulating evidence supports the role of this complex in maintaining translational

62 fidelity through tRNA modifications. Specifically, Elongator is essential for the formation of 5-

63 methoxy-carbonylmethyl (mcm5) and 5-car-bamoylmethyl (ncm5) groups on uridine nucleosides

64 present at the wobble position of many tRNAs $(15,16)$. 
65 Several loss-of-function studies have demonstrated the key role of Elongator during development.

66 Yeast Elp mutants are hypersensitive to high temperature and osmotic conditions and they showed

67 defects in exocytosis, telomeric gene silencing, DNA damage response and adaption to new growth

68 medium $(10,17,18)$. In Arabidopsis thaliana, mutations in Elp subunits resulted in impaired root

69 growth (19) and deletion of Elp3 in Drosophila melanogaster was lethal at the larval stage (20).

70 Depletion of Elongator in Caenorhabditis elegans led to defects in neurodevelopment (21). In

71 mice, Elp1 knockout results in embryonic lethality due to failed neurulation and vascular system

72 formation $(3,22)$. Consistent with its crucial role during development, several human

73 neurodevelopmental disorders have been associated with mutations in Elongator complex

74 subunits. Familial dysautonomia (FD) is caused by a splicing mutation in ELP1 (23-25) that

75 reduces the amount of functional protein in a tissue specific manner, missense mutations in ELP2

76 have been found in individuals with severe intellectual disability (ID) $(26,27)$, variants of ELP3

77 have been associated with amyotrophic lateral sclerosis (ALS) (28), ELP4 variants have been

78 implicated in autism spectrum disorder and Rolandic epilepsy (RE) $(29,30)$ and a mutation in Elp6

79 causes Purkinjie neuron degeneration and ataxia-like phenotypes in mice (31).

80 FD is a neurodevelopmental disorder characterized by widespread sensory and autonomic

81 dysfunction and by central nervous system (CNS) pathology (32-37). The major mutation in FD

82 is a splicing mutation in ELP1 intron 20 that leads to variable skipping of exon 20 and to a

83 reduction of ELP1 mostly in the nervous system $(25,38)$. In 2009 we generated a knockout (KO)

84 Elp1 mouse, Elp1 ${ }^{-/}$and showed that complete ablation of Elp1 resulted in early embryonic lethality

$85(3,22)$. To gain a better understanding how reduction of ELP1 leads to FD, we generated two new

86 mouse models by introducing different copy numbers of the human ELP1 transgene with the major

87 FD mutation (39), $\operatorname{TgFD1}$ and $\operatorname{TgFD9}$, into the Elp1 $1^{-/-}$mouse. Although the human FD transgene 
88 did not rescue embryonic lethality of the Elp1 $1^{-/}$mouse, its expression rescues embryonic

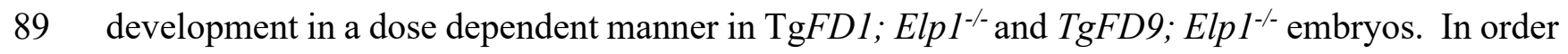

90 to understand the gene regulatory networks that are dependent on ELP1 expression, we conducted

91 a comprehensive transcriptome analysis in these mouse embryos.

93 Results

94 Generation of mice expressing an increasing amount of ELP1.

95 We previously demonstrated that homozygous deletion of the mouse Elp1 gene leads to embryonic

96 lethality prior to mid-gestation (3). Detailed phenotypic characterization of Elp1 ${ }^{-/-} \mathrm{KO}$ embryos at

97 early developmental stages revealed several abnormalities, including a dramatic reduction in size,

98 disruption of the extraembryonic vascular networks, failure of germ layer inversion, and

99 interruption of cephalic neural-tube closure $(3,22)$. In an effort to understand the molecular

100 mechanisms that characterize FD we have generated several transgenic mouse lines carrying the

101 wild-type (WT) and FD human ELP1 gene that differ by the copy number of the transgene (39).

102 The murine Elp1 protein is 80\% identical to human ELP1 and by introducing the human WT ELP1

103 transgene into the Elp1 $1^{-/-}$mouse, we completely rescued development and mice were born alive

104 and healthy, confirming ELP1 functional conservation between human and mouse (3). To test

105 whether the abnormalities caused by ablation of mouse Elpl could be improved by the human FD

106 transgene, heterozygote mice carrying different copy numbers of the FD ELP1 transgene ( $T g F D 1$;

$107 E_{\text {Elp }}^{+/-}$or $T g F D 9 ; E l p 1^{+/}$) were crossed with mice heterozygous for the Elp1 knockout allele

$108\left(\right.$ Elpl $\left.^{-/+}\right)$. Mice were collected at either E8.5 or P0 and genotyped using genomic DNA from the

109 visceral yolk sac. Although neither $T g F D 1$ or $T g F D 9$ rescued embryonic lethality in the Elp1 ${ }^{-/-}$

110 mice (Table 1), the development of the FD1/KO (TgFD1; Elp1 $\left.1^{-/}\right)$and FD9/KO (TgFD9; Elp1 $\left.1^{-/-}\right)$ 
111 embryos progressed further as human ELP1 expression increased (Figure 1a). Because familial

112 dysautonomia is caused by a developmental reduction of ELP1 protein, we conducted a

113 comprehensive transcriptome analysis in embryos expressing increasing amounts of ELP1. We

114 collected 29 individual C57BL/6 mouse embryos at E8.5 ( $\mathrm{n}=8 \mathrm{KO}, \mathrm{n}=7 \mathrm{FD} 1 / \mathrm{KO}, \mathrm{n}=6 \mathrm{FD} 9 / \mathrm{KO}$,

$115 \mathrm{n}=8 \mathrm{WT}$ ) and total RNA was extracted from each single embryo (Figure 1a). As expected, KO

116 embryos do not express any WT Elpl (Figure 1b); whereas FD1/KO and FD9/KO embryos

117 expressed increasing amounts of WT ELP1 with FD9/KO embryos expressing three times more

118 ELP1 than FD1/KO embryos (Figure 1c). Normalized gene count comparisons revealed that the

119 expression of the human ELP1 in FD1/KO embryos is $~ 6 \%$ of WT Elpl while in the FD9/KO the

120 human ELP1 is $\sim 16 \%$ WT Elp1. Principal component analysis revealed that the four genotypes

121 exhibited distinct expression profiles with PC1 explaining $28 \%$ of the total expression variance

122 across samples suggesting that ELP1 dosage plays a critical role in embryonic transcriptome

123 regulation (Figure 1d).

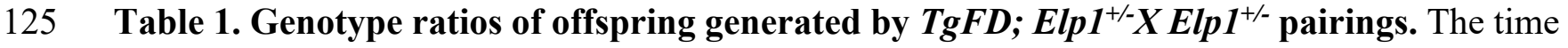

126 of conception is estimated to be E0.5 day prior to the observation of a vaginal plug. P0, postnatal

127 day zero. Expected mendelian ratio is indicated below the genotype, percentage of embryos of

128 each genotype in parentheses.

\begin{tabular}{|c|c|c|c|c|c|c|c|c|}
\hline \multirow{2}{*}{$\begin{array}{c}\operatorname{TgFD} ; \operatorname{Elpl}^{+/-} \\
X \\
{\text { Elp } 1^{+-}}^{-/}\end{array}$} & \multirow{2}{*}{ Time } & \multicolumn{6}{|c|}{ Number of animals } & \multirow{2}{*}{ Total } \\
\hline & & $\begin{array}{l}E_{\text {Elp }} 1^{+/+} \\
12.5 \%\end{array}$ & $\begin{array}{c}E^{E l p 1^{+/}} \\
25 \%\end{array}$ & $\begin{array}{l}\text { Elp1 } 1^{-/} \\
12.5 \%\end{array}$ & $\begin{array}{c}T g ; E l p 1^{+/+} \\
12.5 \%\end{array}$ & $\begin{array}{c}\mathrm{Tg} ; \mathrm{Elpl}^{+/} \\
25 \%\end{array}$ & $\begin{array}{c}\text { Tg; Elp1 } 1^{-/} \\
12.5 \%\end{array}$ & \\
\hline$T g F D 1$ & E8.5 & $17(11 \%)$ & $37(25 \%)$ & $19(13 \%)$ & $17(11 \%)$ & $43(29 \%)$ & $17(11 \%)$ & 150 \\
\hline$T g F D 1$ & P0 & $43(19 \%)$ & $88(39 \%)$ & $0(0 \%)$ & $29(13 \%)$ & $63(28 \%)$ & $0(0 \%)$ & 223 \\
\hline$T g F D 9$ & E8.5 & $10(7 \%)$ & $30(21 \%)$ & $16(11 \%)$ & $25(17 \%)$ & $45(31 \%)$ & $17(11 \%)$ & 143 \\
\hline
\end{tabular}




\begin{tabular}{|c|c|c|c|c|c|c|c|c|}
\hline TgFD9 & P0 & $24(23 \%)$ & $42(40 \%)$ & $0(0 \%)$ & $13(12 \%)$ & $27(25 \%)$ & $0(0 \%)$ & 106 \\
\hline
\end{tabular}

Major transcriptome changes in Elp1 KO embryos.

131 Transcriptome profiling in embryos expressing an increasing amount of ELP1 show that the

132 number of differentially expressed genes (DEGs), (False Discovery Rate or FDR < 0.1; and Fold

133 Change or $\mathrm{FC}>=1.5$ ), proportionally declines as ELP1 expression increases. In KO embryos 2,399

134 out of 19,619 (12.23\%) genes were differentially expressed when compared with WT embryos

135 (Figure 2a, Supplementary Table 1). Strikingly, in FD1/KO embryos the DEGs were only 601

136 (3.06\%), while in FD9/KO embryos there were 494 DEGs (2.52\%) (Figure 2a, Supplementary

137 Table 1), demonstrating that a minimal increase in ELP1 is sufficient to rescue the expression of

$138 \sim 80 \%$ of all DEGs. Gene Ontology $(\mathrm{GO})$ analysis of the down-regulated genes $(\mathrm{FDR}<0.1)$ in $\mathrm{KO}$,

139 FD1/KO and FD9/KO embryos highlighted pathways important for nervous system development

140 including synapse formation, neuron projection and axon growth (Figure 2b, Supplementary Table

141 2). These findings are consistent with the body of work supporting the role of ELPI during early

142 development in target tissue innervation and with the fact that neuronal loss in FD is mostly due

143 to failure of innervation (14, 40-45). Notably, of the 71 genes that were significantly

144 downregulated in all three KO genotypes (Supplementary Table 1), 24 of them ( 33\%) have a

145 critical role in nervous system or brain development (Figure 2c). STRING analysis of these genes

146 revealed an enrichment for protein-protein interactions (PPI enrichment $=2.9 \mathrm{E}-4$ according to

147 STRING v11) (Figure 2c) (46). Among the neuronal genes, Dbxl and Nr2el were the two most

148 down-regulated genes across all three KO genotypes (Figure 2d and 2e). Dbxl, also known as

149 Developing Brain Homeobox 1, is expressed in a regionally restricted pattern in the developing

150 mouse central nervous system (CNS) and encodes for a transcription factor that plays a pivotal

151 role in interneuron differentiation in the ventral spinal cord (47). In vertebrates, spinal interneurons 
152 modulate the motor neuron activity elicited by incoming sensory information and, by relaying the

153 proprioceptive data to the brain, play a critical role in locomotor coordination (48). $\mathrm{Nr} 2 e \mathrm{l}$ is a

154 transcription factor that regulates the expression of genes essential for retinal development (49).

155 Loss of Nr2el in mice has been shown to cause severe early onset retinal degeneration with death

156 of various retinal cells including retinal ganglion cells (RGCs) (50-52). Interestingly, the list of

157 neuronal genes that were significantly downregulated in all three $\mathrm{KO}$ genotypes, $\mathrm{KO}, \mathrm{FD} 1 / \mathrm{KO}$ and

158 FD9/KO, also included the brain-derived neurotrophic factor BDNF-receptor TrkB (Ntrk2), the

159 chemorepulsive axon guidance protein draxin, the homeobox protein involved in brain and sensory

160 organ development otx2, and the neuronal adhesion protein involved in neurite growth neurocan

161 (Ncan) (Figure 2f-i). GO pathways associated with up-regulated genes in the KO embryos are

162 included in Supplementary Figure S1and Supplementary Table 2.

164 Long neuronal genes require Elongator activity for their expression.

165 Although Elongator plays a number of roles in the cell, in the nucleus this complex directly

166 interacts with RNAPII and facilitates transcriptional elongation through altering chromatin

167 structure $(7,10,12,53)$. Elongator has histone acetyltransferase activity via its ELP3 subunit and

168 regulates the accessibility of RNAPII to the chromatin. Using Chromatin immunoprecipitation

169 (ChIP) we have previously shown that in the absence of ELP1, histone H3 acetylation was

170 significantly reduced at the 3' ends of genes (3). Moreover, Close et al demonstrated that upon

171 reduction of ELP1 there was progressively lower RNAPII density at the 3' end of target genes than

172 in the promoter region (14), supporting the role of Elongator in transcriptional elongation. To

173 examine if the downregulation in gene expression observed in the $\mathrm{KO}$ embryos might be due to a

174 failure in transcriptional elongation, we compared the length distribution of the DEGs among 
175 embryos expressing an increasing amount of ELP1. We discovered that long genes, especially

176 those longer than $100 \mathrm{~kb}$, were downregulated significantly more often in the $\mathrm{KO}$ embryos than in

177 the FD9/KO embryos (FDR $<0.01$, Figure 3a) suggesting that Elongator loss has a more

178 pronounced effect on the expression of longer genes. Importantly, ELP1 restoration efficiently

179 rescued their expression. In contrast, there was no difference in gene length in the upregulated

180 genes between the different genotypes (Figure 3b). Of the 257 long genes $(>100 \mathrm{~kb})$ that were

181 downregulated in KO embryos, 216 (84.05\%) were rescued in the FD1/KO embryos and 247

$182(96.11 \%)$ were rescued in FD9/KO embryos. GO analysis of these genes highlighted pathways

183 important for synapse formation, neuron projection and axon growth (Figure 3c, Supplementary

184 Table 3). Since long downregulated genes were enriched for pathways important for nervous

185 system development, we compared the average length of neurodevelopmental genes with the

186 average length of all expressed genes (see Material and Methods) and we observed that

187 neurodevelopmental genes (GO:0048666) were significantly longer (Figure 3d). We then

188 investigated whether the ELP1-dependent gene regulation was driven by length or if neuronal

189 genes were more likely to require ELP1 for efficient transcription. We divided all downregulated

190 genes in $\mathrm{KO}$ embryos into four gene-length categories and examined the proportion of

191 neurodevelopmental genes (GO:0048666) in each category (Figure 3e left panel). If

192 neurodevelopmental genes were more likely to require ELP1 for efficient transcription, we would

193 expect to see more rescue with higher ELP1 dosage. Given that the proportion of

194 neurodevelopmental genes rescued in the FD9/KO embryos in all gene-length categories was

195 similar to those of the downregulated genes in the $\mathrm{KO}$ embryos (Figure 3e right panel), we

196 concluded that neurodevelopmental genes are more susceptible to ELP1-loss simply because they

197 are longer than the non-neurodevelopmental genes. 
199 Identification of genes whose expression correlates with amount of ELP1.

200 Elongator has been linked to transcriptional regulation $(13,14,54)$. Our unique mouse models

201 provide, for the first time, the ability to perform a comprehensive transcriptome analysis to identify

202 genes whose expression depends on the amount of ELP1. This study is highly relevant to better

203 understanding FD pathogenesis, as the disease is caused by a reduction, not loss, of ELP1 primarily

204 in the nervous system. We built the gene co-expression network across embryonic RNASeq data

205 for all genotypes (Materials and Methods). We identified thirty-five co-expression distinct

206 Modules Eigengenes (MEs) (Figure 4a). We then postulated that genes whose expression relies on

207 ELP1 expression would be grouped into three major categories (Figure 4b): (1) genes whose

208 expression changes as a monotonic function of ELP1, referred as "dose-responsive genes"; (2)

209 genes whose expression is completely rescued with low ELP1 expression, referred as "highly

210 responsive genes" and (3) genes whose expression is restored only when ELPl is expressed at WT

211 levels, referred as "low responsive genes". Among the thirty-five MEs identified, ME3, ME2 and

212 M12 had the highest positive correlation with these hypothesized gene patterns (Pearson

213 correlation $>=0.85$, FDR $<0.1$, Figure 4c). ME3 included the dose-responsive genes (Figure 4d);

214 ME2 included the highly responsive genes (Figure 4g) and M12 contained the low responsive

215 genes (Figure 4j). Interestingly, ME4, ME7 and ME10 had the highest negative correlation with

216 our hypothesized patterns (Supplementary Figure S2).

217 Overall, we identified 242 dose-responsive genes whose expression strictly increases as a 218 monotonic function of ELPl (Pearson correlation $>=0.85$, FDR $<0.1$, Figure 4e). The GO

219 analysis of these genes highlighted pathways important for axon and cell projection formation

220 (Figure 4f, Supplementary Table 4). This result supports, once again, the role of ELP1 in the 
221 expression of genes important for target tissue innervation and is consistent with the innervation

222 failure observed in FD (14, 40-45). We found 357 highly responsive genes whose expression was 223 completely restored in $F D 9 / K O$ embryos (Pearson correlation $>=0.85$, FDR $<0.1$, Figure $4 \mathrm{~h}$ ). GO

224 analysis of these genes highlighted pathways associated with transcriptional regulation (Figure 4i,

225 Supplementary Table 4) suggesting that a small increase of functional Elongator is enough to

226 restore normal expression of important transcriptional regulators. This finding might explain the

227 dramatic phenotypic improvement observed in $F D 9 / K O$ embryos when compared with $F D 1 / K O$

228 and KO. Finally, we identified four low responsive genes whose expression was rescued solely in

229 WT embryos: Amigo1, Snn, Unc119b and Sh3pxd2b (Figure 4k) (Pearson correlation $>=0.85$,

$230 \quad$ FDR $<0.1)$.

231 In the negatively correlated patterns ME4, ME7 and ME10 (Pearson correlation $>=0.85$, FDR $<$

232 0.1) we did not find any enrichment for neuronal terms (Supplementary Figure S2, Supplementary

233 Table 5). Genes in ME4 highlighted pathways involved in mitochondrial and respiratory chain

234 activity (Supplementary Figure S3, Supplementary Table 5) while genes in ME7 and ME10 did

235 not shown any significant enrichment for GO terms (FDR $<0.1$ ). These results show that the

236 expression of many genes that are involved in transcriptional regulation and nervous system

237 development positively correlate with ELP1 expression.

239 Discussion

240 Variants in Elongator subunits are associated with various human neurodevelopmental disorders,

241 including FD (ELP1), ID (ELP2), ALS (ELP3), autism spectrum disorder and Rolandic epilepsy

242 (ELP4) (23-31). To gain a better understanding the pathogenesis of FD, we generated two new

243 mouse models expressing an increasing amount of ELP1, FD1/KO and FD9/KO. Although the 
244 human FD transgene did not rescue embryonic lethality in the ELP1 KO mouse, its expression 245 improved embryonic development in a dose dependent manner. To identify genes and pathways 246 whose expression is highly correlated with ELPI and that are ultimately essential for embryonic 247 development, we conducted a comprehensive transcriptome analysis in KO, FD1/KO, FD9/KO 248 and WT embryos. We found that even a minimal increase in ELP1 has a dramatic effect on overall 249 gene expression with the majority of the KO DEGs being completely rescued in the FD1/KO 250 embryos, which only expresses an amount of ELP1 that is approximately $6 \%$ of the Elpl amount 251 expressed in the WT embryos. A significant portion of the down-regulated genes across the 252 different genotypes $\mathrm{KO}, \mathrm{FD} 1 / \mathrm{KO}$ and $\mathrm{FD} 9 / \mathrm{KO}$, have a crucial role in nervous system 253 development. Among these neuronal genes, Dbx1 and Nr2el were the two most down-regulated 254 genes in all three genotypes with absent or reduced expression of ELP1.

255 Dbx 1, also known as Developing Brain Homeobox 1, is expressed in a regionally restricted pattern 256 in the developing mouse central nervous system (CNS) and encodes for a transcription factor that 257 has pivotal role in interneuron differentiation in the ventral spinal cord (47). The spatially restricted 258 expression of $\mathrm{Dbxl}$ is important to establish the distinction of $\mathrm{V} 0$ neurons from the adjacent set of

259 V1 neurons (55). Dbxl mutant mice lacking these ventral interneurons exhibit marked changes in 260 motor coordination, supporting the role of Dbx1-dependent interneurons as key components of the 261 spinal locomotor circuits that control stepping movements in mammals (48). Interestingly, one of 262 the most characteristic features of FD is poor locomotor coordination and both patients as well as 263 a phenotypic mouse model of FD showed progressive impairment in coordination that leads to 264 severe gait ataxia (56-58).

$265 \mathrm{Nr} 2 \mathrm{el}$, or nuclear receptor subfamily 2 group $\mathrm{E}$ member 1, encodes a highly conserved 266 transcription factor known to be a key stem cell fate determinant in both the developing mouse 
267 forebrain and retina $(52,59-63)$. The mouse $N r 2 e 1$ gene is first detected at embryonic day 8 (E8)

268 in the ventricular zone (VZ) of the neuroepithelium layer and later spreads posteriorly into the 269 diencephalon (E10.5) (63-66). In the developing mouse eye, Nr2el is detected in the optic 270 processes of the developing embryo as early as E9 (32), suggesting a role for this gene in an early 271 phase of retinogenesis $(29,61)$. During development, $N r 2 e 1$ null mice display an increase in 272 apoptotic levels of RGCs in the ganglion cell layer (GCL), which results in a marked reduction in 273 thickness of the distinct layers in the adult retina and optic nerve dystrophy $(51,52,66)$. 274 Intriguingly, degeneration of RGCs is observed in two different Elpl conditional knock-out mice 275 (67, 68). In addition, patients with FD show RGCs loss with reduction in the thickness of the retinal 276 nerve fiber layer (RNFL) and progressive vision loss $(33,34,69)$. Although further studies will be 277 necessary to determine the link between ELPI reduction and downregulation of specific key 278 neurodevelopmental genes, the identified ELP1-dependent transcriptome profiles constitute an 279 excellent foundational resource for understanding Elongator biology and also help to shed light 280 into the molecular pathways that underlie diseases caused by disruption of Elongator activity.

281 In the nucleus, Elongator facilitates transcriptional elongation through altering chromatin structure $282(3,7,10,53)$. Therefore, we analyzed the length distribution of DEGs among embryos expressing 283 different ELP1 amounts. Our data clearly showed that long genes are more affected by the loss of 284 functional Elongator compared with shorter genes and, by gradually increasing ELP1 amount, we 285 were able to progressively restore their expression. Moreover, the observation that neuronal genes 286 are significantly longer than all expressed genes, offers a possible explanation about why the 287 nervous system is the tissue that most relies on functional Elongator during embryonic 288 development. In the future it would be interesting to investigate the role of ELPI in transcriptional 289 elongation for each identified target, in order to identify direct regulatory effects. 
290 Several genes that require Elongator to be efficiently expressed have been identified using either

291 cell lines or conditional KO mouse tissues with reduced Elpl expression (14, 45, 70-73). In

292 addition, RNA microarray analysis in post-mortem FD tissues has shown that a subset of genes

293 involved in myelination require ELP1 for efficient transcription (74). In the current study we have

294 identified gene patterns whose expression varies as a function of ELP1 amount: (1) genes whose

295 expression changes as a monotonic function of $E L P 1$; (2) genes whose expression is completely

296 restored with low amount of ELP1 and (3) genes whose expression is restored only when ELPI is

297 expressed at WT levels (Figure 4c). It is interesting that distinctive groups of genes display

298 different Elongator dependence. Genes important for axon formation and cell projection responded

299 in a dose-dependent manner to functional Elongator amounts. This is consistent with the

300 observation that, even though Elongator is a ubiquitously expressed protein complex, variants

301 affecting different Elongator subunits all lead to neurodevelopmental diseases (23-31). On the

302 other hand, the expression of important transcriptional regulators is already restored in $F D 9 / K O$

303 embryos, suggesting that only a small increase of functional Elongator is necessary to rescue their

304 expression. This might underlie the dramatic phenotypic improvement observed in $F D 9 / K O$

305 embryos when compared with $F D 1 / K O$ and $\mathrm{KO}$. We then identified sets of genes whose expression

306 negatively correlated with ELP1. GO analysis of these genes highlighted pathways involved in

307 mitochondrial and respiratory chain activity.

308 In conclusion, our study is the first to assess the in vivo dose-dependent effect of ELPI in early

309 development using transcriptome analysis. We demonstrated that even a minimal increase in

310 ELP1 can have a dramatic effect on both mouse embryonic development and global gene

311 expression. Although loss of ELPl compromised the expression of a large number of genes, this

312 study shows that neuronal genes are more sensitive to ELPl reduction. We recognize that further 
313 studies will be necessary to determine which of the individual gene expression changes are direct

314 versus indirect. However, the identification of gene networks and biological pathways that are

315 regulated by ELP1-dosage is highly relevant to better understand the pathogenesis of FD, as well

316 as other neurodevelopmental diseases caused by Elongator deficiency. The data presented here

317 will help to identify potential biomarkers for future clinical studies and new targetable pathways

318 for therapy.

320 Materials and Methods

321 Generation of $F D 1 / K O, F D 9 / K O$ mouse models and genotyping

322 The detailed description of the original strategy to generate the Elp1 knockout mouse line has been

323 previously published (3). A detailed description of the generation the $\operatorname{Tg} F D$ transgenic lines

324 carrying different copy number of the human ELPI gene with the IVS20+6T $>$ C mutation can be

325 found in our previous manuscript by Hims et al. (39). To create the TgFD1; Elp1 ${ }^{-/}$and $\operatorname{TgFD9}$;

326 Elp1 $1^{-/}$mouse, we crossed the previously generated $\operatorname{TgFD} 1$ or $\operatorname{TgFD} 9$ transgenic mouse line with

327 the mouse line heterozygous for the null allele $E l p 1^{-/+}$. The resulting progeny was genotyped to

328 detect the presence of the $T g F D 1$ or $T g F D 9$ transgene and of the null allele $E l p 1^{-}$. As expected,

329 the $T g F D 1$ and $T g F D 9$ transgene segregated independently from the null allele; therefore, around

330 one-fourth of the F1 mice carried both the $T g F D$ transgene and null Elp1 alleles (TgFD1; Elp1 $1^{+/-}$

331 or $\left.T g F D 9 ; E l p 1^{+/}\right)$. Subsequently, we crossed the TgFD1; Elp1 ${ }^{+/}$and $T g F D 9 ; E l p 1^{+/-}$mice with

332 the mouse line heterozygous for the null Elp1 allele $\left(E l p 1^{+/}\right)$. The resulting progeny was genotyped

333 to detect the presence of the TgFD1 or TgFD9 transgene as well as the null allele in homozygosis

$334\left(E l p 1^{-/}\right)$. The Elp1 $1^{-/}, T g F D 1 ; E l p 1^{-/}, T g F D 9 ; E l p 1^{-/}$and $E l p 1^{+/+}$embryos were produced by

335 crossing heterozygote mice carrying different copy numbers of the FD ELP1 transgene (TgFD1; 
$336 E l p 1^{+/-}$or $\operatorname{TgFD9}$; Elp1 $\left.1^{+/}\right)$with heterozygote mice $\left(E l p 1^{-/+}\right)$. The day of vaginal-plug discovery

337 was designated E 0.5 . The mice used for this study were housed in the animal facility of

338 Massachusetts General Hospital (Boston, MA), provided with constant access to a standard diet of

339 food and water, maintained on a 12-hour light/dark cycle, and all experimental protocols were

340 approved by the Subcommittee on Research Animal Care at the Massachusetts General Hospital.

341 The genotypes of animals and embryos were determined by PCR analysis of genomic DNA from

342 tail biopses and from embryos and/or visceral yolk sacs, respectively. The primer sets used were

343 as follows: for determining the wild-type Elp1 allele, 5'-ACCCTCAGGCAGTTTGATTG-3' and

344 5'-CATGGCTCCATAAAACAAACAC-3'; for detecting the knockout allele,

345 5'ACCCTCAGGCAGTTTGATTG-3' and 5'-GGCTACCGGCTAAAACTTGA-3'; and

346 for determining the human TgFD transgenes, TgProbe1F 5'-GCCATTGTACTGTTTGCGACT-

347 3' and TgProbe1R 5'-TGAGTGTCACGATTCTTTCTGC-3'.

349 Morphological analysis of embryos

350 Photographs of visceral yolk sacs and embryos were taken with a digital camera Leica DFC7000

351 T mounted on a Leica M205 FCA dissection microscope. LAS X software (Leica) was used for

352 image processing.

\section{RNA Seq experiment}

355 RNA was extracted from $8 \mathrm{Elpl}^{-/}, 7 \mathrm{TgFD1}$; $\mathrm{Elpl}^{-/}, 6 \mathrm{TgFD} 9 ; \mathrm{Elpl}^{-/}$and $8 \mathrm{Elpl}^{+/+}$individual

356 embryos at the embryonic stage of E8.5 using the QIAzol Reagent and following the

357 manufacturer's instructions. RNAseq Libraries were prepared using TruSeq ${ }^{\circledR}$ Stranded mRNA

358 Library Prep kit (Illumina 20020594), using 100 ng total RNA as input. Libraries were evaluated 
359 for final concentration and size distribution by Agilent 2200 TapeStation and/or qPCR, using

360 Library Quantification Kit (KK4854, Kapa Biosystems), and multiplexed by pooling equimolar

361 amounts of each library prior to sequencing. Pooled libraries were 50 base pair paired-end

362 sequenced on Illumina HiSeq 2500 across multiple lanes. Real time image analysis and base

363 calling were performed on the HiSeq 2500 instrument using HiSeq Sequencing Control Software

364 (HCS) and FASTQ files demultiplexed using CASAVA software version 1.8.

365 A synthesized transcriptome reference was generated, by artificially adding the sequence of human

366 ELP1 gene from the Ensembl human transcriptome reference GRCh37.75 to the Ensembl mouse

367 transcriptome reference GRCm38.83 as an independent chromosome. RNASeq reads were

368 mapped to this synthesized transcriptome reference 3 by STAR v2.5.2b, allowing only uniquely

369 mapped reads with 5\% mismatch (75).

\section{Differential gene expression analysis}

372 Gene counts were generated by HTSeq-count v0.11.2 with "-s reverse" option to be compatible

373 with Illumina TruSeq reads, according to the gene annotations of the synthesized transcriptome

374 reference. Four genotypes were defined as "FD0", "FD1", "FD9" and WT to reflect the amounts

375 of human and mouse ELP1. Gene counts across samples were filtered so that only genes whose

376 median expression amounts were no less than 0.1 counts-per-million-reads in at least one genotype

377 were kept for the downstream analysis. The R (v3.6.1) package "SVA" v3.32.1 was implemented

378 on the filtered gene expression matrix to estimate surrogated variables (SVs) among samples. A

379 generalized linear model was built by the R package "DESeq2" v1.24.0 to correlated gene

380 expression to genotypes reflecting human ELP1 and mouse Elp1 amounts, together with all the

381 estimated SVs. During the differential gene expression analysis, the FC and FDR of each genotype 
382 was estimated per gene. PCA analysis was implemented based on the top 500 most variable genes

383 across samples, from the filtered gene expression matrix with SVs corrected.

\section{GO analysis}

386 GO analysis was done by GOrilla (76) (http://cbl-gorilla.cs.technion.ac.il/). Organism was set as

387 Mus musculus. Two unranked lists of genes were used for each GO analysis. The GO analysis for

388 DEGs used the following two lists: 1) the list of DEGs for a genotype and 2) the list of all genes

389 in the filtered gene expression matrix. The GO analysis for co-expression modules used the

390 following two lists: 1) the list of genes highly correlated with a hypothetical pattern in a co-

391 expression module and 2) the list of all genes in the filtered gene expression matrix. In the figures

392 related to the GO analysis for DEGs, ten significant terms in the ontology of Cellular Component

393 were selected to be plotted in Figure 2b. The complete list of DEG GO terms for each genotype

394 can be found in Supplementary Table 2.

\section{Mouse neuronal genes}

397 Mouse neuronal genes were extracted and downloaded via AmiGO2 from geneontology.org, using

398 the key word 'neuron' and restricting organism as 'Mus musculus'. Totally 3,442 unique neuronal

399 genes were found.

\section{Co-expression modules analysis}

402 R package "WGCNA" (77) v1.68 was implemented to the filtered gene expression matrix with

403 SVs corrected. The soft-thresholding power was determined to be 5. The minimal module size was

404 set to 30 . The raw modules were merged using a dis-similarity cutoff of 0.15 . 


\section{Correlation between co-expression modules and hypothetical patterns}

407 Dummy expression data were generated to mimic three hypothetical patterns, namely the

408 monotonic increase pattern, the monotonic decrease pattern, and the saturated pattern. The eigen

409 vector representing each co-expression module was then correlated with each of the hypothetical

410 patterns using Pearson correlation.

\section{Statistical Analysis}

413 All raw $\mathrm{p}$ values in this study, if multiple tests were involved, were corrected by the Benjamini-

414 Hochberg Procedure and converted to FDR values. Wald test was applied in the differential gene 415 expression analysis. A significant DEG of a genotype, compared to WT, was defined as FDR <

4160.1 and the $\mathrm{FC}>=1.5$ for that genotype. Fisher's exact test was used for GO analysis, where a 417 significant enrichment for a GO term was defined as FDR $<0.1$. Kolmogorov-Smirnov test (K-S 418 test) was applied to compare the gene length distribution of genes from different groups. A 419 significant difference in length distribution between two groups was defined as FDR $<0.1$. A 420 significant correlation throughout this study was defined as the Pearson correlation coefficient $>=$ 4210.85 and FDR $<0.1$. For all box plots, the middle lines inside boxes indicated the medians. The 422 lower and upper hinges corresponded to the first and third quartiles. Each box extended to 1.5 423 times inter-quartile range (IQR) from upper and lower hinges respectively. The symbols *, ** and $424 * * *$, if appeared in the figures, indicated $\mathrm{FDR}<0.1,<0.01$ and $<0.001$, respectively. 
427 We thank Dr. Lucy Norcliffe-Kaufmann and Dr. Horacio Kaufmann of the Dysautonomia

428 Treatment and Evaluation Center at New York University Medical School for their long-standing

429 collaboration and helpful discussions. We are also grateful to Dr. Frances Lefcort for her

430 comments on the manuscript. This work was supported by National Institutes of Health (NIH)

431 grants (R37NS095640 to S.A.S.), the Francis Crick Institute (Cancer Research UK FC001166 to

432 PC and JQS), the UK Medical Research Council (FC001166 to PC and JQS) and the Welcome

433 Trust (FC001166 to PC and JQS).

\section{Competing interests}

436 The authors declare competing financial interests.

437 Funding: Research support from PTC Therapeutics, Inc. (S.A.S.).

438 Personal financial interests: Susan A. Slaugenhaupt is a paid consultant to PTC Therapeutics and

439 is an inventor on several U.S. and foreign patents and patent applications assigned to the

440 Massachusetts General Hospital, including U.S Patents 8,729,025 and 9,265,766, both entitled

441 "Methods for altering mRNA splicing and treating familial dysautonomia by administering

442 benzyladenine," filed on August 31, 2012 and May 19, 2014 and related to use of kinetin; and U.S.

443 Patent 10,675,475 entitled, “Compounds for improving mRNA splicing” filed on July 14, 2017

444 and related to use of BPN-15477. Elisabetta Morini, Dadi Gao, Michael E. Talkowski and Susan

445 A. Slaugenhaupt are inventors on an International Patent Application Number

446 PCT/US2021/012103, assigned to Massachusetts General Hospital and entitled " RNA Splicing

447 Modulation" related to use of BPN-15477 in modulating splicing. 


\section{Referances}

450 1. Li F, Lu J, Han Q, Zhang G, Huang B. The Elp3 subunit of human Elongator complex is

451 functionally similar to its counterpart in yeast. Mol Genet Genomics. 2005;273(3):264-72.

452 2. Chen Z, Zhang H, Jablonowski D, Zhou X, Ren X, Hong X, et al. Mutations in

$453 \mathrm{ABO}$ /ELO2, a subunit of holo-Elongator, increase abscisic acid sensitivity and drought tolerance

454 in Arabidopsis thaliana. Mol Cell Biol. 2006;26(18):6902-12.

455 3. Chen YT, Hims MM, Shetty RS, Mull J, Liu L, Leyne M, et al. Loss of mouse Ikbkap, a

456 subunit of elongator, leads to transcriptional deficits and embryonic lethality that can be rescued

457 by human IKBKAP. Mol Cell Biol. 2009;29(3):736-44.

458 4. Fellows J, Erdjument-Bromage H, Tempst P, Svejstrup JQ. The Elp2 subunit of elongator

459 and elongating RNA polymerase II holoenzyme is a WD40 repeat protein. J Biol Chem.

$460 \quad 2000 ; 275(17): 12896-9$.

4615 5inkler GS, Petrakis TG, Ethelberg S, Tokunaga M, Erdjument-Bromage H, Tempst P, et

462 al. RNA polymerase II elongator holoenzyme is composed of two discrete subcomplexes. J Biol

463 Chem. 2001;276(35):32743-9.

464 6. Frohloff F, Jablonowski D, Fichtner L, Schaffrath R. Subunit communications crucial for

465 the functional integrity of the yeast RNA polymerase II elongator (gamma-toxin target (TOT))

466 complex. J Biol Chem. 2003;278(2):956-61.

467 7. Otero G, Fellows J, Li Y, de Bizemont T, Dirac AM, Gustafsson CM, et al. Elongator, a 468 multisubunit component of a novel RNA polymerase II holoenzyme for transcriptional elongation. 469 Mol Cell. 1999;3(1):109-18. 
470 8. Creppe C, Malinouskaya L, Volvert ML, Gillard M, Close P, Malaise O, et al. Elongator

471 controls the migration and differentiation of cortical neurons through acetylation of alpha-tubulin.

472 Cell. 2009;136(3):551-64.

473 9. Tran HT, Nimick M, Uhrig RG, Templeton G, Morrice N, Gourlay R, et al. Arabidopsis

474 thaliana histone deacetylase 14 (HDA14) is an alpha-tubulin deacetylase that associates with PP2A

475 and enriches in the microtubule fraction with the putative histone acetyltransferase ELP3. Plant J. $476 \quad 2012 ; 71(2): 263-72$.

477 10. Wittschieben BO, Otero G, de Bizemont T, Fellows J, Erdjument-Bromage H, Ohba R, et 478 al. A novel histone acetyltransferase is an integral subunit of elongating RNA polymerase II 479 holoenzyme. Mol Cell. 1999;4(1):123-8.

480 11. Pokholok DK, Harbison CT, Levine S, Cole M, Hannett NM, Lee TI, et al. Genome-wide 481 map of nucleosome acetylation and methylation in yeast. Cell. 2005;122(4):517-27.

482 12. Kim JH, Lane WS, Reinberg D. Human Elongator facilitates RNA polymerase II 483 transcription through chromatin. Proc Natl Acad Sci U S A. 2002;99(3):1241-6.

484 13. Han Q, Hou X, Su D, Pan L, Duan J, Cui L, et al. hELP3 subunit of the Elongator complex 485 regulates the transcription of HSP70 gene in human cells. Acta Biochim Biophys Sin (Shanghai). $486 \quad 2007 ; 39(6): 453-61$.

487 14. Close P, Hawkes N, Cornez I, Creppe C, Lambert CA, Rogister B, et al. Transcription 488 impairment and cell migration defects in elongator-depleted cells: implication for familial 489 dysautonomia. Mol Cell. 2006;22(4):521-31.

490 15. Huang B, Johansson MJ, Bystrom AS. An early step in wobble uridine tRNA modification 491 requires the Elongator complex. RNA. 2005;11(4):424-36. 
492 16. Fichtner L, Frohloff F, Jablonowski D, Stark MJ, Schaffrath R. Protein interactions within

493 Saccharomyces cerevisiae Elongator, a complex essential for Kluyveromyces lactis zymocicity.

494 Mol Microbiol. 2002;45(3):817-26.

495 17. Rahl PB, Chen CZ, Collins RN. Elp1p, the yeast homolog of the FD disease syndrome 496 protein, negatively regulates exocytosis independently of transcriptional elongation. Mol Cell. $497 \quad 2005 ; 17(6): 841-53$.

498 18. Li Q, Fazly AM, Zhou H, Huang S, Zhang Z, Stillman B. The elongator complex interacts 499 with PCNA and modulates transcriptional silencing and sensitivity to DNA damage agents. PLoS 500 Genet. 2009;5(10):e1000684.

501 19. Nelissen H, Fleury D, Bruno L, Robles P, De Veylder L, Traas J, et al. The elongata 502 mutants identify a functional Elongator complex in plants with a role in cell proliferation during 503 organ growth. Proc Natl Acad Sci U S A. 2005;102(21):7754-9.

504 20. Walker J, Kwon SY, Badenhorst P, East P, McNeill H, Svejstrup JQ. Role of elongator 505 subunit Elp3 in Drosophila melanogaster larval development and immunity. Genetics. $506 \quad 2011 ; 187(4): 1067-75$.

507 21. Solinger JA, Paolinelli R, Kloss H, Scorza FB, Marchesi S, Sauder U, et al. The 508 Caenorhabditis elegans Elongator complex regulates neuronal alpha-tubulin acetylation. PLoS 509 Genet. 2010;6(1):e1000820.

510 22. Dietrich P, Yue J, E S, Dragatsis I. Deletion of exon 20 of the Familial Dysautonomia gene 511 Ikbkap in mice causes developmental delay, cardiovascular defects, and early embryonic lethality. 512 PLoS One. 2011;6(10):e27015.

513 23. Anderson SL, Coli R, Daly IW, Kichula EA, Rork MJ, Volpi SA, et al. Familial 514 dysautonomia is caused by mutations of the IKAP gene. Am J Hum Genet. 2001;68(3):753-8. 
515 24. Slaugenhaupt SA, Blumenfeld A, Gill SP, Leyne M, Mull J, Cuajungco MP, et al. Tissue-

516 specific expression of a splicing mutation in the IKBKAP gene causes familial dysautonomia. Am

517 J Hum Genet. 2001;68(3):598-605.

518 25. Cuajungco MP, Leyne M, Mull J, Gill SP, Lu W, Zagzag D, et al. Tissue-specific reduction

519 in splicing efficiency of IKBKAP due to the major mutation associated with familial

520 dysautonomia. Am J Hum Genet. 2003;72(3):749-58.

521 26. Najmabadi H, Hu H, Garshasbi M, Zemojtel T, Abedini SS, Chen W, et al. Deep

522 sequencing reveals 50 novel genes for recessive cognitive disorders. Nature. 2011;478(7367):57-

$523 \quad 63$.

524 27. Cohen JS, Srivastava S, Farwell KD, Lu HM, Zeng W, Lu H, et al. ELP2 is a novel gene

525 implicated in neurodevelopmental disabilities. Am J Med Genet A. 2015;167(6):1391-5.

526 28. Simpson CL, Lemmens R, Miskiewicz K, Broom WJ, Hansen VK, van Vught PW, et al.

527 Variants of the elongator protein 3 (ELP3) gene are associated with motor neuron degeneration.

528 Hum Mol Genet. 2009;18(3):472-81.

529 29. Strug LJ, Clarke T, Chiang T, Chien M, Baskurt Z, Li W, et al. Centrotemporal sharp wave

530 EEG trait in rolandic epilepsy maps to Elongator Protein Complex 4 (ELP4). Eur J Hum Genet.

$5312009 ; 17(9): 1171-81$.

532 30. Addis L, Ahn JW, Dobson R, Dixit A, Ogilvie CM, Pinto D, et al. Microdeletions of ELP4

533 Are Associated with Language Impairment, Autism Spectrum Disorder, and Mental Retardation.

534 Hum Mutat. 2015;36(9):842-50.

535 31. Kojic M, Gaik M, Kiska B, Salerno-Kochan A, Hunt S, Tedoldi A, et al. Elongator

536 mutation in mice induces neurodegeneration and ataxia-like behavior. Nature communications.

$5372018 ; 9(1): 3195$. 
538 32. Axelrod FB, Hilz MJ, Berlin D, Yau PL, Javier D, Sweat V, et al. Neuroimaging supports 539 central pathology in familial dysautonomia. J Neurol.257(2):198-206.

540 33. Mendoza-Santiesteban CE, Hedges TR, 3rd, Norcliffe-Kaufmann L, Warren F, Reddy S, 541 Axelrod FB, et al. Clinical neuro-ophthalmic findings in familial dysautonomia. J 542 Neuroophthalmol.32(1):23-6.

543 34. Mendoza-Santiesteban CE, Palma JA, Hedges TR, 3rd, Laver NV, Farhat N, Norcliffe544 Kaufmann L, et al. Pathological Confirmation of Optic Neuropathy in Familial Dysautonomia. J $545 \quad$ Neuropathol Exp Neurol. 2017;76(3):238-44.

546 35. Ochoa JG. Familial dysautonomia (Riley-Day syndrome) may be associated with epilepsy. 547 Epilepsia. 2003;44(3):472.

548 36. Mahloudji M, Brunt PW, McKusick VA. Clinical neurological aspects of familial 549 dysautonomia. J Neurol Sci. 1970;11(4):383-95.

550 37. Pearson J. Familial dysautonomia (a brief review). J Auton Nerv Syst. 1979;1(2):119-26.

551 38. Slaugenhaupt SA. Genetics of familial dysautonomia. Tissue-specific expression of a 552 splicing mutation in the IKBKAP gene. Clin Auton Res. 2002;12 Suppl 1:I15-9.

553 39. Hims MM, Shetty RS, Pickel J, Mull J, Leyne M, Liu L, et al. A humanized IKBKAP 554 transgenic mouse models a tissue-specific human splicing defect. Genomics. 2007;90(3):389-96.

555 40. Ohlen SB, Russell ML, Brownstein MJ, Lefcort F. BGP-15 prevents the death of neurons 556 in a mouse model of familial dysautonomia. Proc Natl Acad Sci U S A. 2017;114(19):5035-40.

557 41. George L, Chaverra M, Wolfe L, Thorne J, Close-Davis M, Eibs A, et al. Familial 558 dysautonomia model reveals Ikbkap deletion causes apoptosis of Pax3+ progenitors and peripheral 559 neurons. Proc Natl Acad Sci U S A. 2013;110(46):18698-703. 
560 42. Jackson MZ, Gruner KA, Qin C, Tourtellotte WG. A neuron autonomous role for the

561 familial dysautonomia gene ELP1 in sympathetic and sensory target tissue innervation.

562 Development. 2014;141(12):2452-61.

563 43. Cheishvili D, Maayan C, Cohen-Kupiec R, Lefler S, Weil M, Ast G, et al. IKAP/Elp1

564 involvement in cytoskeleton regulation and implication for familial dysautonomia. Hum Mol

565 Genet. 2011;20(8):1585-94.

566 44. Johansen LD, Naumanen T, Knudsen A, Westerlund N, Gromova I, Junttila M, et al. IKAP

567 localizes to membrane ruffles with filamin A and regulates actin cytoskeleton organization and 568 cell migration. J Cell Sci. 2008;121(Pt 6):854-64.

569 45. Abashidze A, Gold V, Anavi Y, Greenspan H, Weil M. Involvement of IKAP in peripheral 570 target innervation and in specific JNK and NGF signaling in developing PNS neurons. PLoS One. $571 \quad 2014 ; 9(11): \mathrm{e} 113428$.

572 46. Szklarczyk D, Gable AL, Lyon D, Junge A, Wyder S, Huerta-Cepas J, et al. STRING v11: 573 protein-protein association networks with increased coverage, supporting functional discovery in 574 genome-wide experimental datasets. Nucleic Acids Res. 2019;47(D1):D607-D13.

575 47. Lu S, Shashikant CS, Ruddle FH. Separate cis-acting elements determine the expression 576 of mouse Dbx gene in multiple spatial domains of the central nervous system. Mech Dev. 577 1996;58(1-2):193-202.

578 48. Lanuza GM, Gosgnach S, Pierani A, Jessell TM, Goulding M. Genetic identification of 579 spinal interneurons that coordinate left-right locomotor activity necessary for walking movements. 580 Neuron. 2004;42(3):375-86. 
581 49. Yu RT, Chiang MY, Tanabe T, Kobayashi M, Yasuda K, Evans RM, et al. The orphan 582 nuclear receptor Tlx regulates Pax2 and is essential for vision. Proc Natl Acad Sci U S A. $5832000 ; 97(6): 2621-5$.

584 50. Abrahams BS, Kwok MC, Trinh E, Budaghzadeh S, Hossain SM, Simpson EM.

585 Pathological aggression in "fierce" mice corrected by human nuclear receptor 2E1. J Neurosci. $586 \quad 2005 ; 25(27): 6263-70$.

587 51. Zhang CL, Zou Y, Yu RT, Gage FH, Evans RM. Nuclear receptor TLX prevents retinal 588 dystrophy and recruits the corepressor atrophin1. Genes Dev. 2006;20(10):1308-20.

589 52. Miyawaki T, Uemura A, Dezawa M, Yu RT, Ide C, Nishikawa S, et al. Tlx, an orphan 590 nuclear receptor, regulates cell numbers and astrocyte development in the developing retina. J $591 \quad$ Neurosci. 2004;24(37):8124-34.

592 53. Hawkes NA, Otero G, Winkler GS, Marshall N, Dahmus ME, Krappmann D, et al. 593 Purification and characterization of the human elongator complex. J Biol Chem. $594 \quad 2002 ; 277(4): 3047-52$.

595 54. Li F, Ma J, Ma Y, Hu Y, Tian S, White RE, et al. hElp3 directly modulates the expression 596 of HSP70 gene in HeLa cells via HAT activity. PLoS One. 2011;6(12):e29303.

597 55. Pierani A, Moran-Rivard L, Sunshine MJ, Littman DR, Goulding M, Jessell TM. Control 598 of interneuron fate in the developing spinal cord by the progenitor homeodomain protein Dbx1. 599 Neuron. 2001;29(2):367-84.

600 56. Morini E, Gao D, Montgomery CM, Salani M, Mazzasette C, Krussig TA, et al. ELP1 601 Splicing Correction Reverses Proprioceptive Sensory Loss in Familial Dysautonomia. Am J Hum 602 Genet. 2019. 
603 57. Macefield VG, Norcliffe-Kaufmann L, Gutierrez J, Axelrod FB, Kaufmann H. Can loss of

604 muscle spindle afferents explain the ataxic gait in Riley-Day syndrome? Brain. 2011;134(Pt

$605 \quad 11): 3198-208$.

606 58. Macefield VG, Norcliffe-Kaufmann LJ, Axelrod FB, Kaufmann H. Relationship between

607 proprioception at the knee joint and gait ataxia in HSAN III. Mov Disord. 2013;28(6):823-7.

608 59. Schmouth JF, Banks KG, Mathelier A, Gregory-Evans CY, Castellarin M, Holt RA, et al.

609 Retina restored and brain abnormalities ameliorated by single-copy knock-in of human NR2E1 in

610 null mice. Mol Cell Biol. 2012;32(7):1296-311.

611 60. Jackson A, Panayiotidis P, Foroni L. The human homologue of the Drosophila tailless gene

612 (TLX): characterization and mapping to a region of common deletion in human lymphoid leukemia

613 on chromosome 6q21. Genomics. 1998;50(1):34-43.

614 61. Li W, Sun G, Yang S, Qu Q, Nakashima K, Shi Y. Nuclear receptor TLX regulates cell

615 cycle progression in neural stem cells of the developing brain. Mol Endocrinol. 2008;22(1):56-64.

616 62. Pignoni F, Baldarelli RM, Steingrimsson E, Diaz RJ, Patapoutian A, Merriam JR, et al.

617 The Drosophila gene tailless is expressed at the embryonic termini and is a member of the steroid

618 receptor superfamily. Cell. 1990;62(1):151-63.

619 63. Yu RT, McKeown M, Evans RM, Umesono K. Relationship between Drosophila gap gene

620 tailless and a vertebrate nuclear receptor Tlx. Nature. 1994;370(6488):375-9.

621 64. Monaghan AP, Grau E, Bock D, Schutz G. The mouse homolog of the orphan nuclear 622 receptor tailless is expressed in the developing forebrain. Development. 1995;121(3):839-53.

623 65. Monaghan AP, Bock D, Gass P, Schwager A, Wolfer DP, Lipp HP, et al. Defective limbic 624 system in mice lacking the tailless gene. Nature. 1997;390(6659):515-7. 
625 66. Young KA, Berry ML, Mahaffey CL, Saionz JR, Hawes NL, Chang B, et al. Fierce: a new 626 mouse deletion of $\mathrm{Nr} 2 \mathrm{e} 1$; violent behaviour and ocular abnormalities are background-dependent.

627 Behav Brain Res. 2002;132(2):145-58.

628 67. Ueki Y, Shchepetkina V, Lefcort F. Retina-specific loss of Ikbkap/Elp1 causes

629 mitochondrial dysfunction that leads to selective retinal ganglion cell degeneration in a mouse 630 model of familial dysautonomia. Dis Model Mech. 2018;11(7).

631 68. Ueki Y, Ramirez G, Salcedo E, Stabio ME, Lefcort F. Loss of Ikbkap Causes Slow,

632 Progressive Retinal Degeneration in a Mouse Model of Familial Dysautonomia. eNeuro. $6332016 ; 3(5)$

634 69. Mendoza-Santiesteban CE, Hedges Iii TR, Norcliffe-Kaufmann L, Axelrod F, Kaufmann 635 H. Selective retinal ganglion cell loss in familial dysautonomia. J Neurol.261(4):702-9.

636 70. Goffena J, Lefcort F, Zhang Y, Lehrmann E, Chaverra M, Felig J, et al. Elongator and 637 codon bias regulate protein levels in mammalian peripheral neurons. Nature communications. $638 \quad 2018 ; 9(1): 889$.

639 71. Zeltner N, Fattahi F, Dubois NC, Saurat N, Lafaille F, Shang L, et al. Capturing the biology 640 of disease severity in a PSC-based model of familial dysautonomia. Nat Med. 2016;22(12):14216417.

642 72. Cohen-Kupiec R, Pasmanik-Chor M, Oron-Karni V, Weil M. Effects of IKAP/hELP1 643 deficiency on gene expression in differentiating neuroblastoma cells: implications for familial 644 dysautonomia. PLoS One.6(4):e19147.

645 73. Boone N, Bergon A, Loriod B, Deveze A, Nguyen C, Axelrod FB, et al. Genome-wide 646 analysis of familial dysautonomia and kinetin target genes with patient olfactory ecto647 mesenchymal stem cells. Hum Mutat.33(3):530-40. 
648 74. Cheishvili D, Maayan C, Smith Y, Ast G, Razin A. IKAP/hELP1 deficiency in the 649 cerebrum of familial dysautonomia patients results in down regulation of genes involved in 650 oligodendrocyte differentiation and in myelination. Hum Mol Genet. 2007;16(17):2097-104.

651 75. Dobin A, Davis CA, Schlesinger F, Drenkow J, Zaleski C, Jha S, et al. STAR: ultrafast 652 universal RNA-seq aligner. Bioinformatics. 2013;29(1):15-21.

653 76. Eden E, Navon R, Steinfeld I, Lipson D, Yakhini Z. GOrilla: a tool for discovery and 654 visualization of enriched GO terms in ranked gene lists. BMC Bioinformatics. 2009;10:48.

655 77. Zhang B, Horvath S. A general framework for weighted gene co-expression network 656 analysis. Stat Appl Genet Mol Biol. 2005;4:Article17. 
a
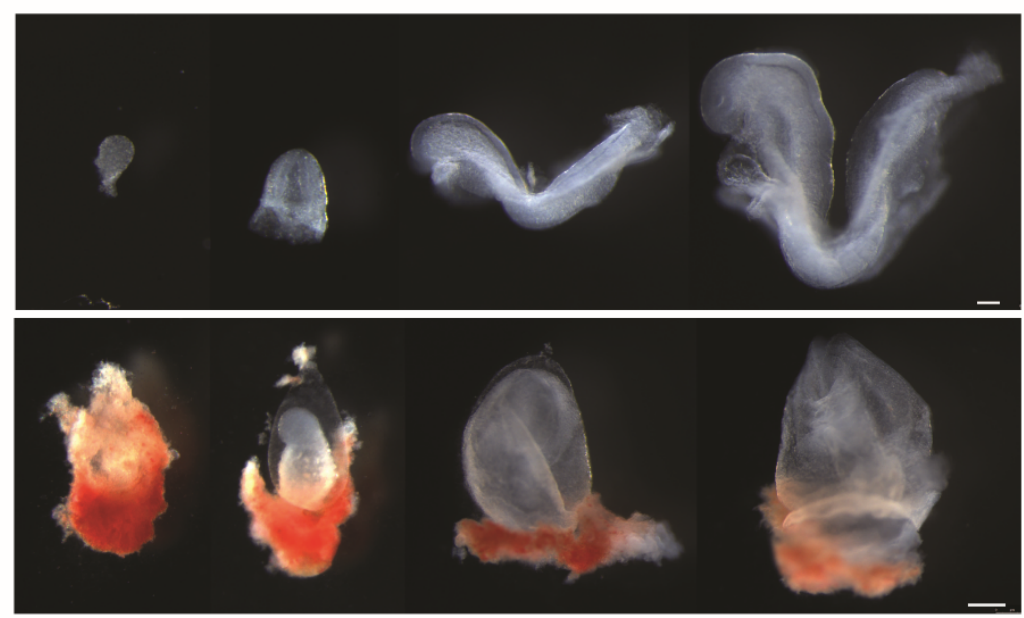

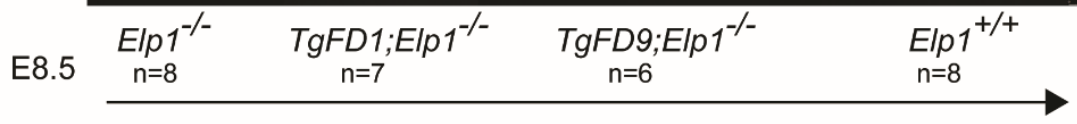

RNA seq in 29 individual embryos

b

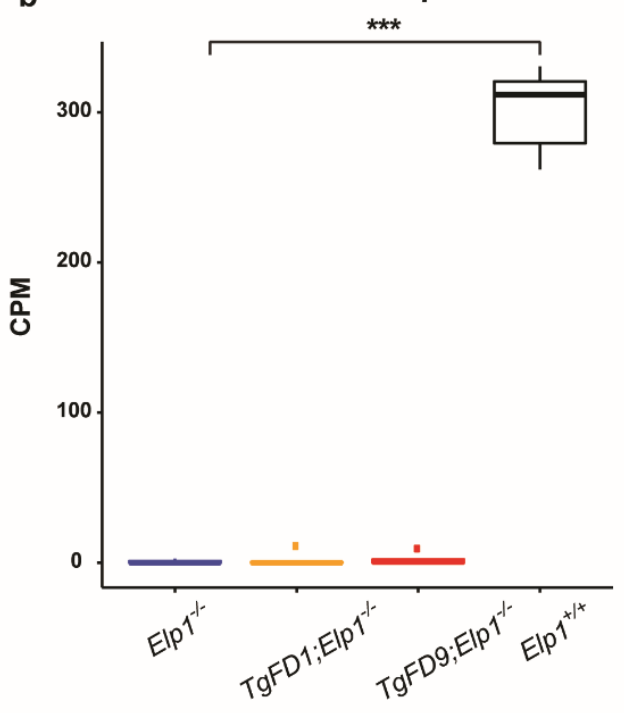

c

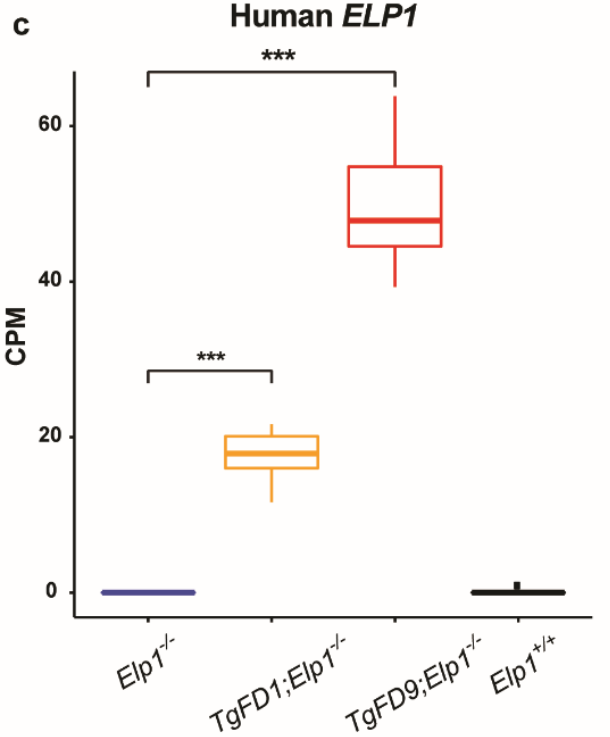

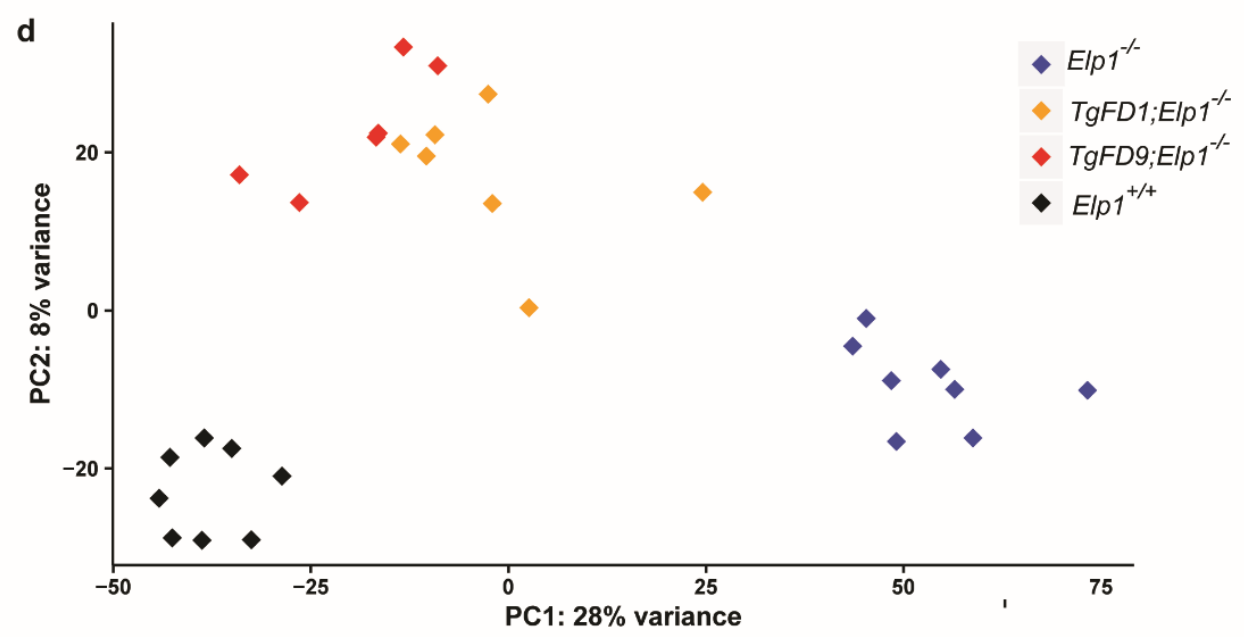


658 Figure 1. Generating mouse embryos expressing increasing amount of ELP1 (a)

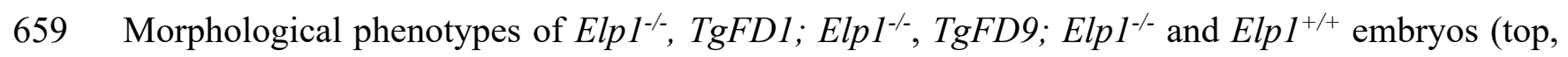

660 the scale bar represents $100 \mu \mathrm{m}$ ) and extraembryonic components (bottom, the scale bar represents

$661250 \mu \mathrm{m})$ at E8.5. RNA-seq experiment was performed using total RNA extracted by individual

662 embryo. (b) Expression of the endogenous WT Elp1 across different embryos. The median for

663 each group is shown. (c) Expression of the human WT ELP1 across different embryos. The median

664 for each group is shown. (d) Principal component analysis of all the embryos colored by genotype.

665 In box-and-whisker plots in b-c, each box extends to 1.5 times inter-quartile range (IQR) from

666 upper and lower hinges, respectively. Outliers are not shown. Only comparisons with significant

667 difference are marked by stars (two-tailed, unpaired Welch's $t$ test with Bonferroni correction). In

668 the figure, $* P<0.05 ; * * P<0.01 ; * * * P<0.001$ 


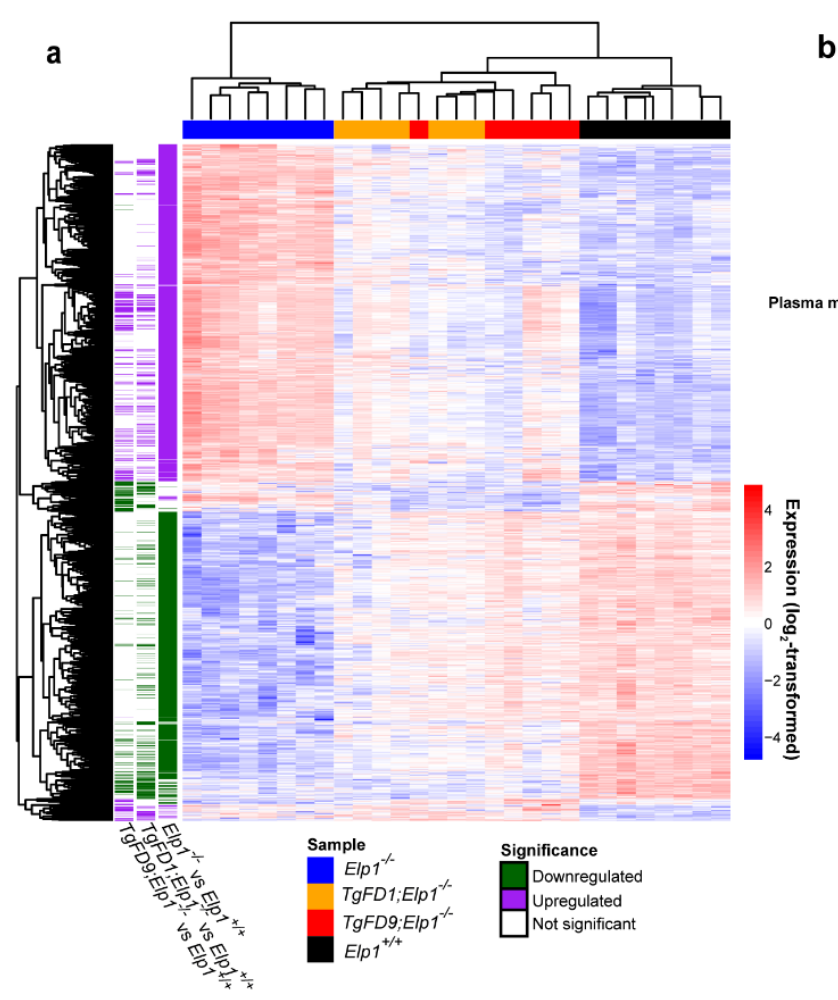

c

Leprel2 Zfp365 Braxin Scg5

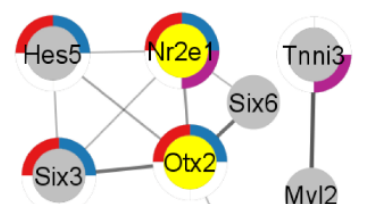

b

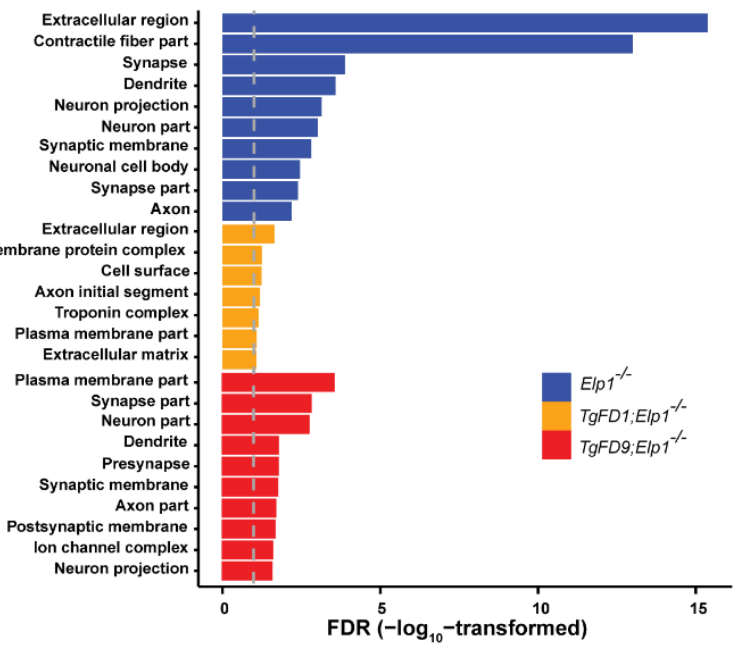

Ms4a4d Dmrta1 Lrtm1 Cpne8

Gm16503 Celf3 mCG_1155Bcas1

Gra3 Lamc3 Hap1 Ryr3 Prmt8 Kenqz Gja5

Jakmip2 Ssc5d Ntrk2 Casq1 Srcin Brsk2 SIc25a48

S1pr3 Lrrn1 Doc2a Gdpd2 Sema6c C1ql1 Nova2
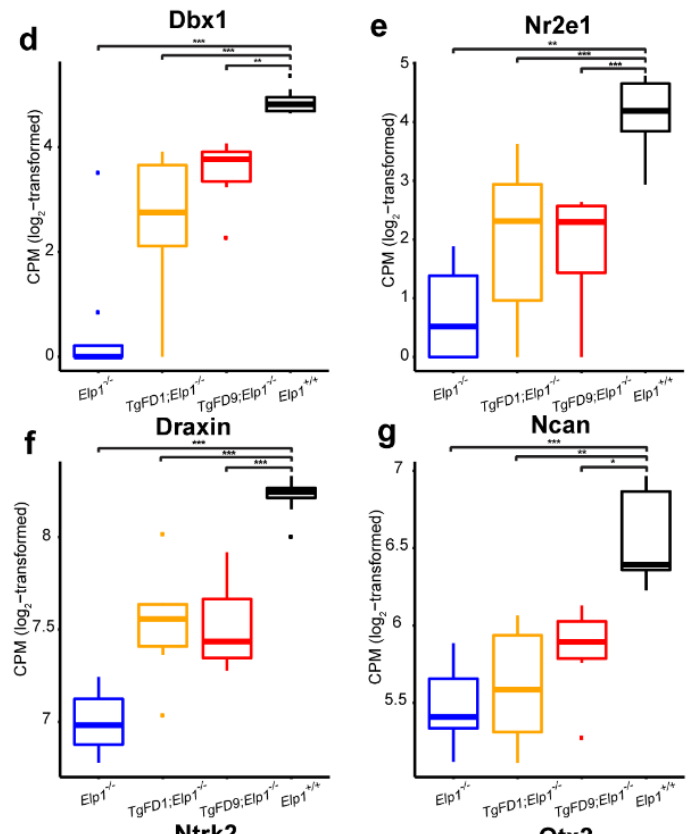

Psd2 Fam69c Iglon5 Ptprn2 Heyl Aldh1a3 Lhfpl4

Adssl1 Ikbkap Ncan Cbr3y Reepz Slc2a4 Trpt1

Emilin3 Mipol1 Prelid2 BC068157 Vgll2 Gdap111 Sorcs1

ENSMUSP00000095870
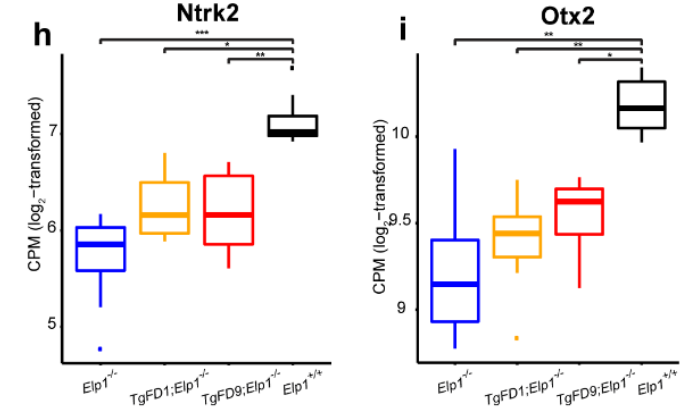

Figure 2. Transcriptome profiling of embryos expressing increasing amount of ELP1. (a)

672 Heatmap of the 2,619 differentially expressed genes for each genotype compared to WT Elp1 $1^{+/+}$.

673 (b) Gene ontology analysis of the downregulated genes in Elp1 $1^{-/}, \operatorname{TgFD1}$; Elp1 $1^{-/}, \operatorname{TgFD9}$; Elp1 $1^{-/-}$ 
674 embryos. The graph shows FDR values for the most significant specific GO terms (see also

675 Supplementary Table 2). (c) STRING interaction map for the 71 genes that were significantly

676 downregulated in all three KO genotypes (see also Supplementary Table 1). Each pie indicates a

677 gene. Note, Ikbkap is the alternative name of Elpl. The pies surrounded by colored edges indicate

678 genes involved in neurogenesis (GO:0022008, blue), brain development (GO:0007420, red),

679 regulation of synapse organization (GO:0050807, green), and nervous system process

680 (GO:0050877, purple), respectively. The pies in yellow indicate the genes whose expression are

681 highlighted in panels d-i. (d-i) box-and-whisker plots showing the expression of key genes for

682 nervous system development that are significantly downregulated across all three KO genotypes:

$683 \operatorname{Dbx} 1(\mathbf{d}), \operatorname{Nr} 2 \mathrm{e} 1(\mathbf{e}), \operatorname{Draxin}(\mathbf{f}), \operatorname{Ncan}(\mathbf{g}), \operatorname{Ntrk} 2(\mathbf{h})$ and Otx2 (i). ${ }^{*} P<0.05, * * P<0.01$ and $* * * P$

$684<0.001$, Welch's $t$ test. 


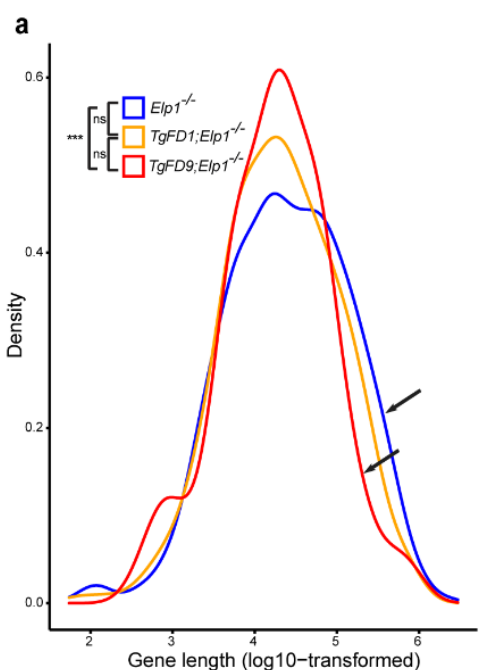

d

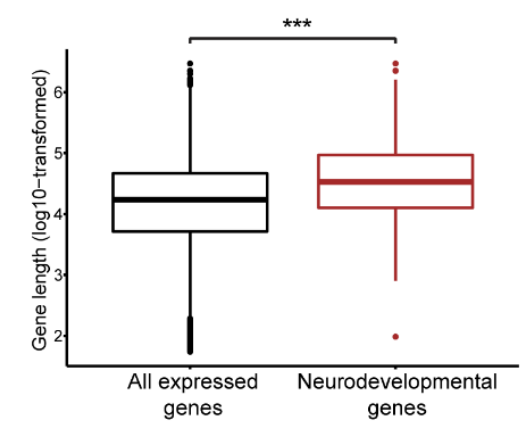

b

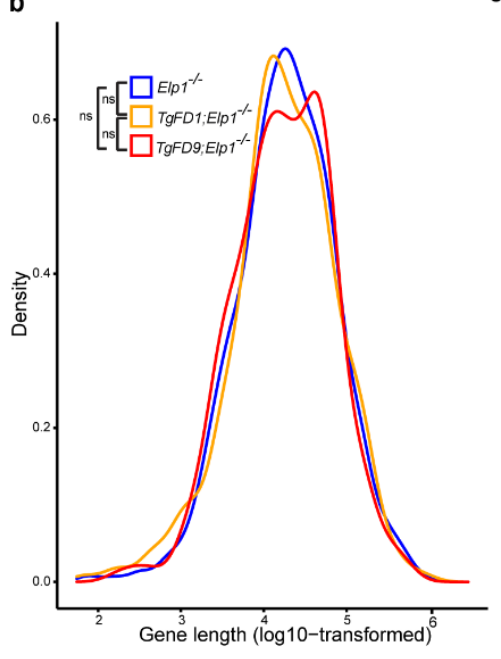

e

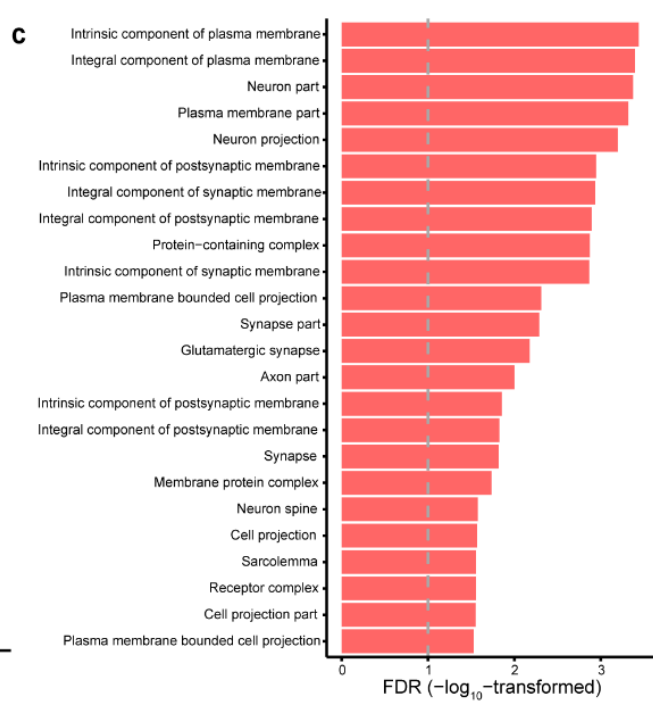

Neurodevelopmental genes All downregulated genes in Elp $1^{-1 /}$ embryos

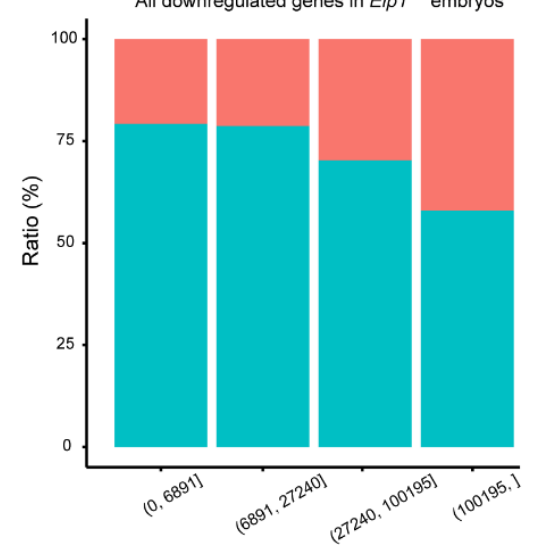

Non-neurodevelopmental genes

Downregulated genes rescued in TgFD9; Elp $1^{-/-}$embryos

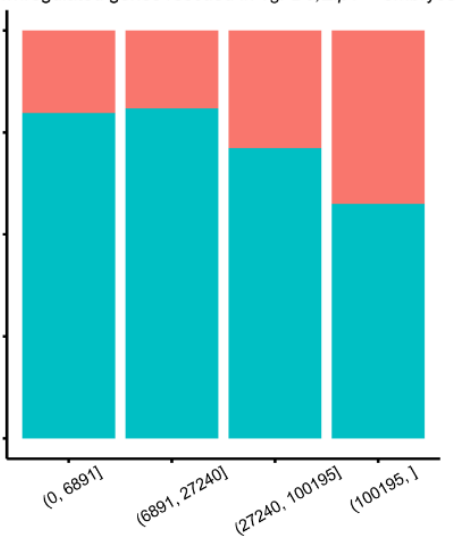

687 Figure 3. Gene length distribution in embryos expressing different ELP1 amounts. (a) Length

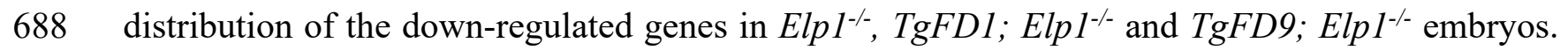

689 Arrows indicate the comparison between Elp1 ${ }^{-/-}$and $\operatorname{TgFD9}$; Elp1 ${ }^{-/}$distribution. (b) Length

690 distribution of the up-regulated genes in Elp1 $1^{-/}, T g F D 1 ; E l p 1^{-/}$and TgFD9; Elp1 ${ }^{-/-}$embryos. In

691 both (a) and (b), K-S test was applied. (c) Gene ontology analysis of the 247 long genes (> 100

$692 \mathrm{~kb}$ ) downregulated in Elp1 $1^{-/}$embryos and rescued at $T g F D 9 ; E l p 1^{-/-}$. The graph shows FDR values

693 for each specific GO term (see also Supplementary Table 3). (d) Comparison between length

694 distributions of all expressed genes in RNASeq with the length distribution of neuronal genes

695 (GO:0048666 in Gene Ontology database) via two-tailed, unpaired Welch's $t$ test. (e) Ratio of 
696 neurodevelopmental and non-neurodevelopmental genes in different gene-length ranges. The left

697 panel represents all downregulated genes in Elp1 $1^{-/}$embryos, while the right panel represents genes

698 which expression was rescued in TgFD9; Elp1 $1^{-/}$embryos. The gene-length ranges were

699 determined in order to have an equal number of genes in each range. In the figure, ns, not

$700 \quad$ significant and $* P<0.05 ; * * P<0.01 ; * * * P<0.001$ 
bioRxiv preprint doi: https://doi.org/10.1101/2021.04.16.440179; this version posted April 16, 2021. The copyright holder for this preprint (which was not certified by peer review) is the author/funder. All rights reserved. No reuse allowed without permission.
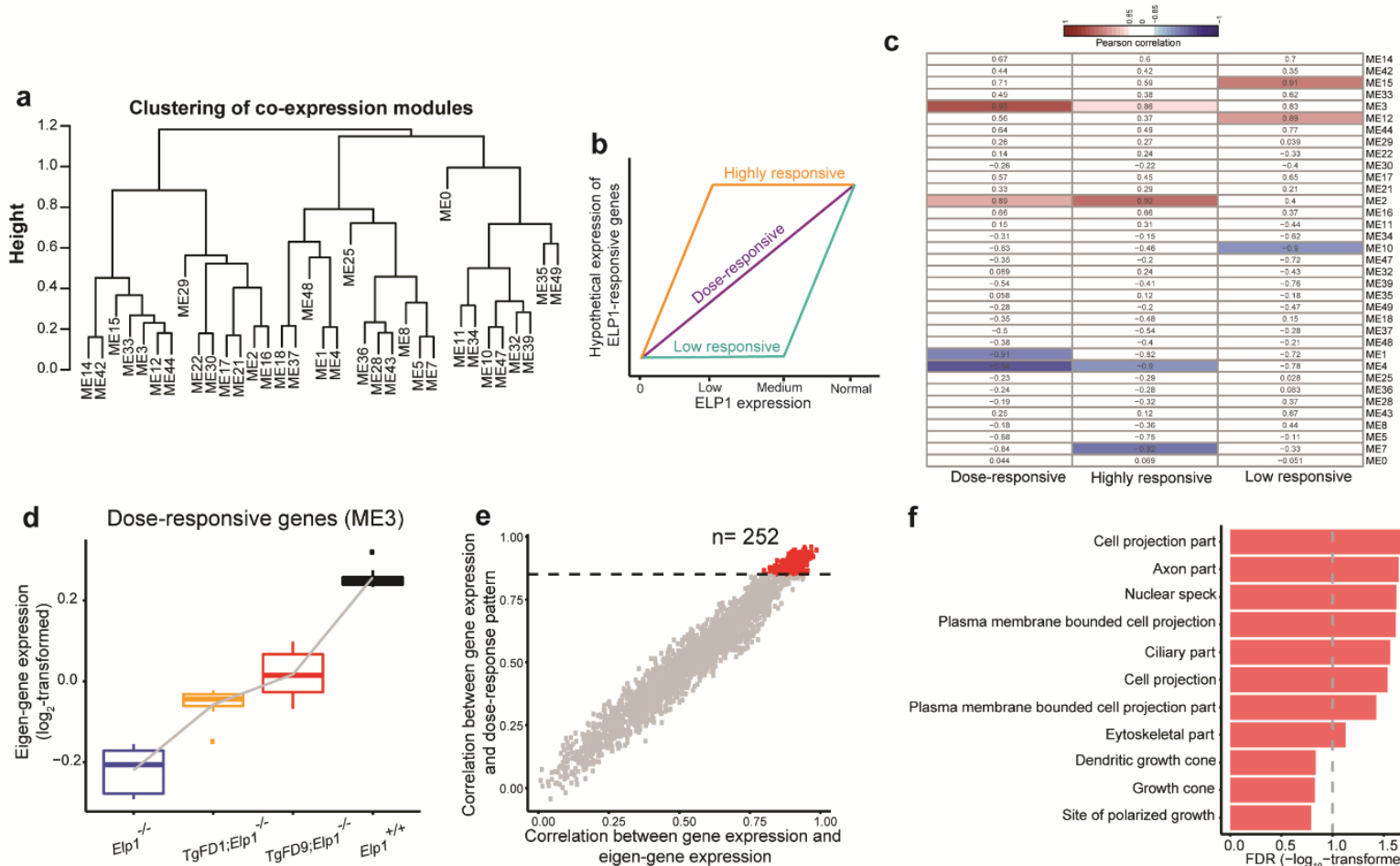

f
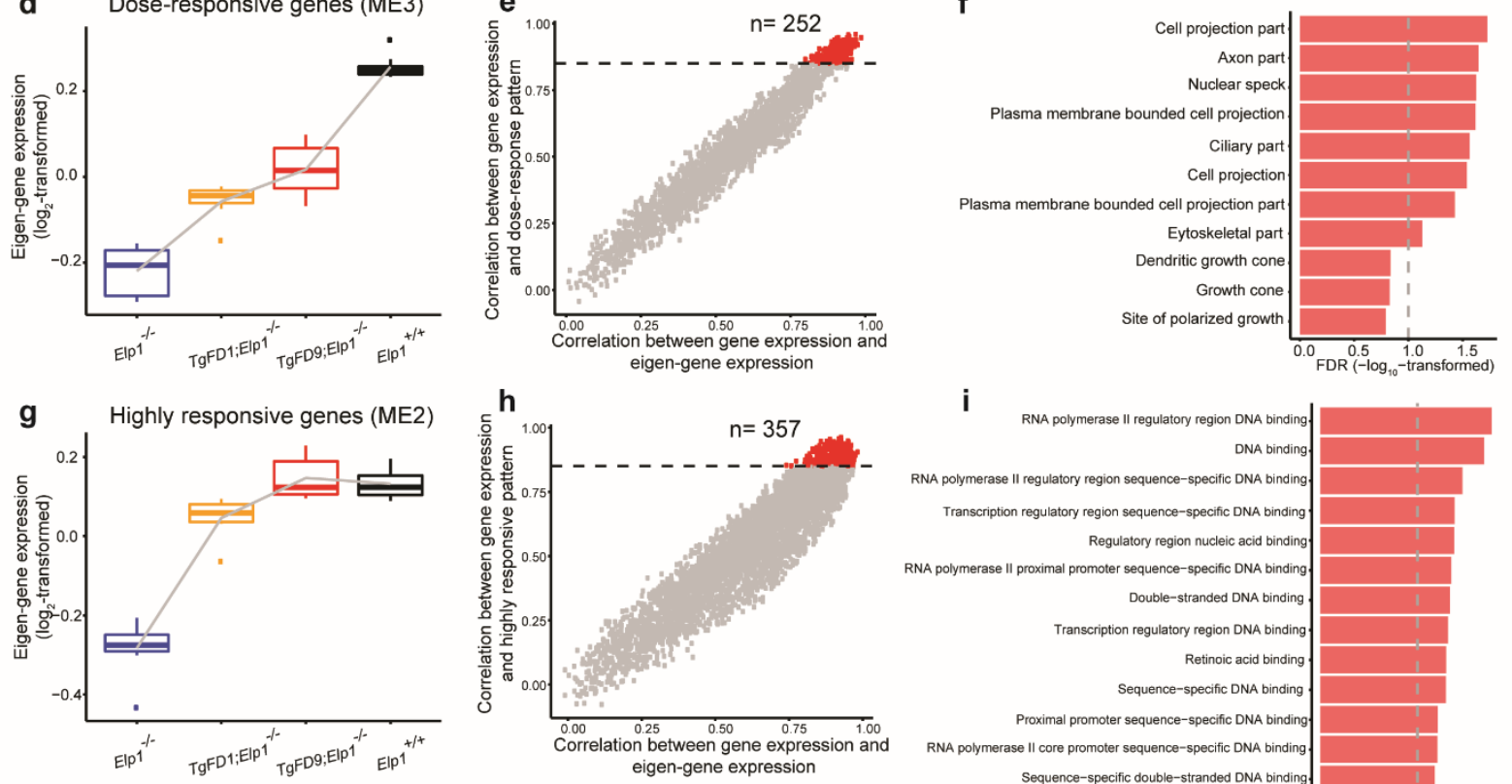

i
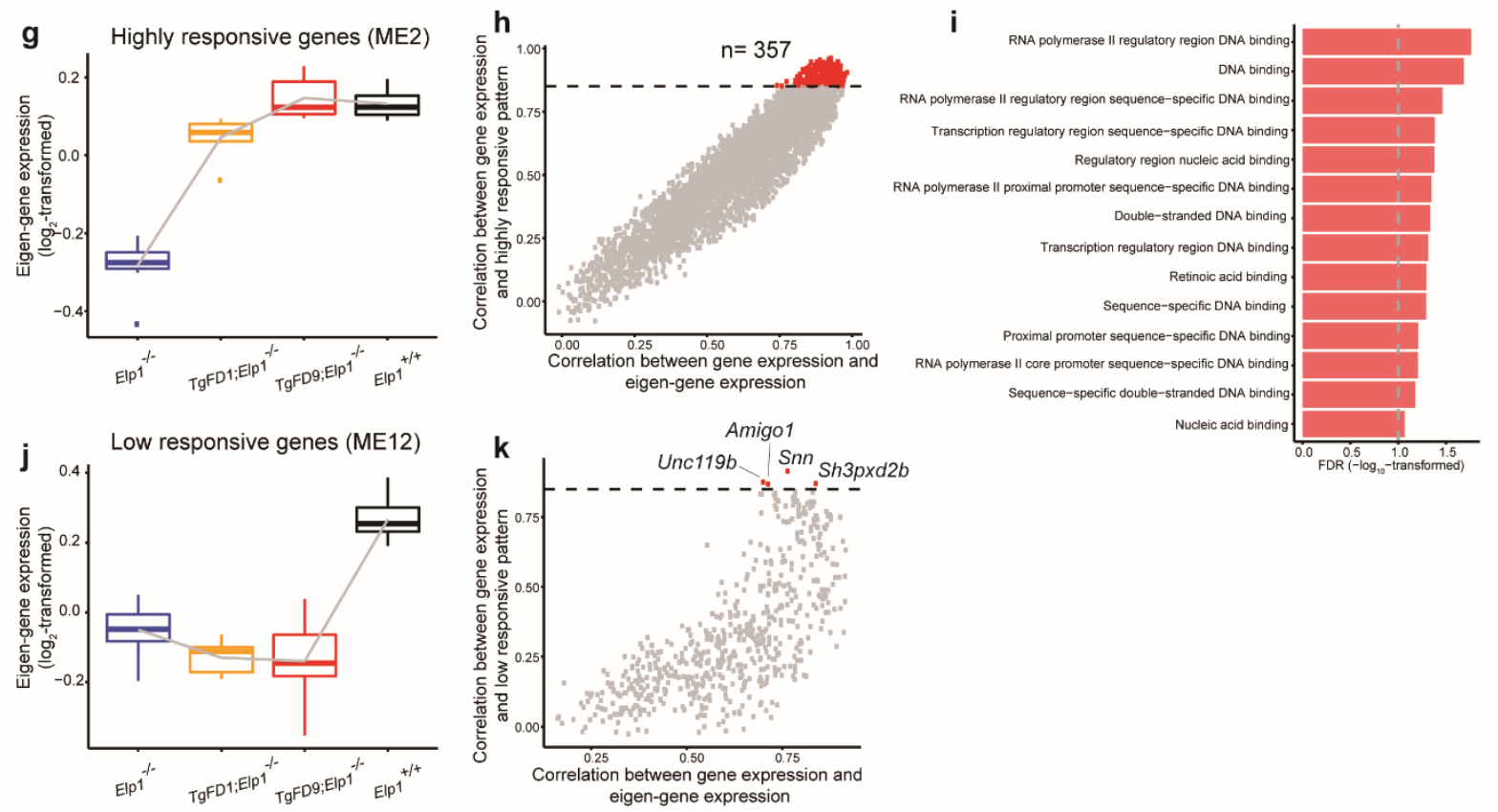
703 Figure 4. Identification of ELP1-responsive gene patterns. (a) Modules of distinct Eigen (ME)

704 analysis across embryos expressing increasing amount of ELP1 identified thirty-five distinct MEs

705 (see also Materials and Methods). (b) Hypothetical expression trajectories of ELP1-responsive

706 genes. (c) Heatmap displays the Pearson correlation between the eigen values of each co-

707 expression module and the eigen values of each hypothetical expression trajectory. The blue

708 domain indicates a negative correlation while the red domain indicates a positive correlation. The

709 number in each grid demonstrates the Pearson correlation coefficient. (d) Boxplot displays the

710 eigen values of gene expression of ME3 at each genotype. (e) Module membership of each gene

711 in ME3. Each dot represents a gene. The $\mathrm{x}$-axis demonstrates the Pearson correlation between gene

712 expression and module eigen values of ME3 while the y-axis demonstrates the Pearson correlation

713 between gene expression and the eigen values of each hypothetical expression trajectory. The

714 horizontal dashed line shows a correlation coefficient of 0.85 . (f) Gene ontology analysis of the

715252 dose-responsive genes which expression strictly increase as monotonic function of $E L P 1$. The

716 graph shows FDR values for each specific GO term (see also Supplementary Table 4). (g) Boxplot

717 displays the eigen values of gene expression of ME2 at each genotype. (h) Module membership

718 of each gene in ME2. (i) Gene ontology analysis of the 357 highly responsive genes whose

719 expression is completely restored in $F D 9 / K O$ embryos. The graph shows FDR values for each

720 specific GO term (see also Supplementary Table 4). (j) Boxplot displays the eigen values of gene

721 expression of ME12 at each genotype. (k) Module membership of each gene in ME12. ${ }^{*} P<0.05$;

$722 * * P<0.01 ; * * * P<0.001$, Welch's $t$ test. 